\title{
Feasibility and Profitability Analysis of Debris Removal Industry Yunyu Shen ${ }^{*}$, Ye Huang, Zhao Li \\ Northeastern University, Shen Yang, R.P.China \\ *Corresponding author Yunyu Shen, Bachelor, 919304858@qq.com
}

\begin{abstract}
This paper explores feasibility of debris removal industry and conducts its profitability analysis. A comprehensive assessment model based on Entropy-weighting TOPSIS method is proposed to assess six most popular debris removal methods and then determine an optimal one. The model combines five measure indexes (cycle, cost, effect, risk and benefit) to evaluate feasibility of each option quantitatively and qualitatively. Further, with help of optimized Monte Carlo method, AHP etc, conclusions are made that Electrodynamics Tether method and satellite are the optimal solution for short term and long term respectively. Last, strength and weakness are discussed while future work is addressed.
\end{abstract}

Key words: entropy-weightingTOPSIS; debris removal industry; profitability analysis comprehensive assessment model; Monte Carlo method; tether method

\section{Introduction}

\subsection{Problem background}

Space debris, a potential threat to space vehicles, has constantly increased over time, which arouses more and more attention. Fortunately, several methods have been raised to remove space debris, such as space-based water jets, laser system and satellites designed for clearing debris etc. An emerging industry is waiting to rise sharply, thus a best option for space debris removal is required.

In sum, we mainly face two following problems. One is that we need to analyze quantitatively or qualitatively each method from cost, risk and benefit etc. The second is that we need to assess the best option or combination of options after reviewing and comparison.

\subsection{Previous research}

With the rapid development of space exploration, many experts begin to pay attention to removal methods for space debris and many research papers related are published.

Determining the best method for debris removal is actually an evaluation problem. Several methods are put forward to solve the problem, one of which is The Technique for Order of Preference by Similarity to Ideal Solution (TOPSIS), originally developed by Hwang and Yoon in 1981. It is a method of compensatory aggregation that compares a set of alternatives by identifying weights for each criterion, normalizing scores for each criterion and calculating the geometric distance between each alternative and the ideal alternative, which is the best score in each criterion. ${ }^{1}$

Generally speaking, in comprehensive evaluation, the greater variation degree for a sort of index value is, the smaller information entropy is, and the larger information content provided by the index is and the greater weight of the index is. Otherwise, the smaller weight 
of the index is. Hence according to the variation degree of each index value, entropy weight, weight of each index, can be calculated by entropy .

Therefore, entropy weight TOPSIS method is adopted here to establish the assessment model.

\section{Experimental results and discussion}

In order to find out the best method, we assess each method from following five aspects, which are cycle, cost, effect, risk, and benefit. Six common methods for debris removal are evaluated by our model, i.e.,

- Satellite: capture the object and remove it;

- CubeSat: propelled by small satellite to capture debris to low earth orbit

- LODR :Laser Orbital Debris Removal

- Electrodynamics Tether: constant current in wire coil makes retired satellite fall down slowly until burning in the atmosphere

- Robot: capture space debris with mechanical arms

- Acquirer: aimed at de-orbit mission (search and clear debris in orbits with $800-1000 \mathrm{~km}$ altitude)

\subsection{Factor 1: cycle}

We introduce the concept of cycle time $(\mathrm{T})$ : we can circularly use mature achievements repeatedly after developing the technology successfully, and length of each use is $T$.

Through considering the relationship between length of cycle time and planned total duration of an operating project, different assessments on different total duration can be got for company to choose a best option according to total duration of planned operation, as shown in Table 1.

Table 1 - Project life cycle

\begin{tabular}{|c|c|c|}
\hline Method & \multicolumn{2}{|c|}{$\begin{array}{c}\text { Relative } \\
\text { coefficient }\end{array}$} \\
\hline LODR & 2 & 0.2083 \\
\hline Satellite & 2 & 0.2083 \\
\hline Acquirer & 1.5 & 0.1562 \\
\hline Robot & 1.8 & 0.1875 \\
\hline $\begin{array}{c}\text { Electrodynamics } \\
\text { Tether }\end{array}$ & 0.8 & 0.0833 \\
\hline CubeSat & 1.5 & 0.1562 \\
\hline
\end{tabular}

\subsection{Factor 2: cost—— optimized monte-carlo method}

Characteristic of Monte Carlo method is that its predicted result provides section range and distribution regularities of predicted value. Predicted result given by this method is more scientific and reasonable. ${ }^{2}$

Precision can be improved and convergence procedure can be prolonged through 
comprehensively utilizing skills to reduce variance. In this model, the random variable will generate through studies on limit sampling shown in Fig. 1 and stratified sampling shown in Fig. 2.

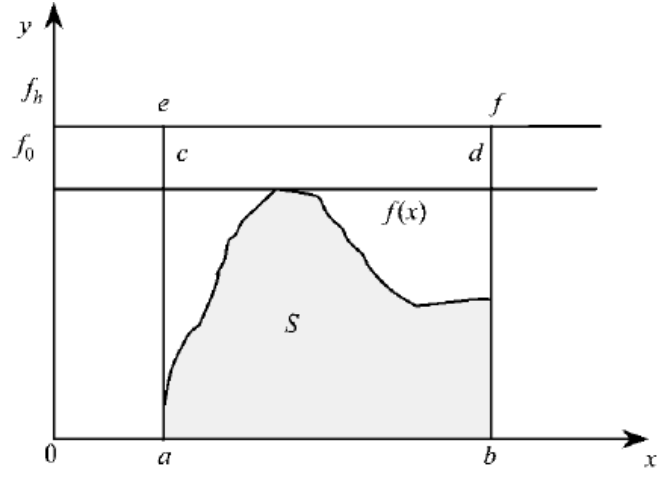

Fig.1 - Limiting sample

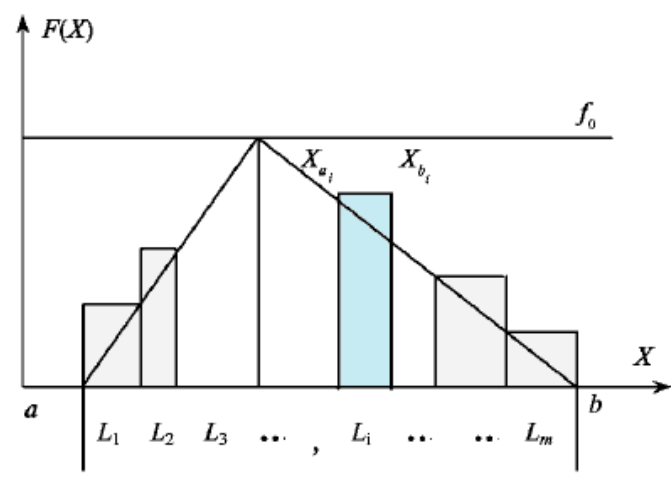

Fig.2 - Delaminating sample

\subsection{Factor 3: effect—analytic hierarchy process(AHP)}

The AHP provides a comprehensive and rational framework for structuring a decision problem, for representing and quantifying its elements, for relating those elements to overall goals, and for evaluating alternative solutions. ${ }^{3}$

Ranking and grade of each evaluation object is shown in the Table 2.

Table 2 - Ranking and grade of each evaluation object

\begin{tabular}{|l|l|l|l|l|l|}
\hline Rank & Name & \multicolumn{2}{|c|}{ Grade } & Rank & Name \\
\hline $\mathbf{1}$ & Satellite & 0.2296 & $\mathbf{4}$ & Acquirer & 0.1456 \\
\hline $\mathbf{2}$ & Cubesats & 0.1927 & $\mathbf{5}$ & LODR & 0.1441 \\
\hline $\mathbf{3}$ & Electrodynamics Tether & 0.1565 & $\mathbf{6}$ & Robo & 0.1316 \\
\hline
\end{tabular}

Therefore, ranking of monocycle removal result is: Satellite, CubeSat, Electrodynamics Tether, Acquirer, LODR and Robot.

$>$ From the aspect of result, the first three factors which play significant role in evaluating removal results of method for space debris are altitude, size and amount of space debris remaining to be removed.

\subsection{Factor 4: risk—_three-dimensional risk matrix model}

A Risk matrix figure originates from risk matrix, it is a valid risk management tool, which can be applied to potential risk analysis for a sort of method.

Risk matrix method comprehensively considers two factors, i.e., risk probability and risk impact. Instead of acquiring judgment matrix from expert opinions directly, the method determine grading of risk impact and risk occurrence probability beforehand, then experts judge quantitative level through intuitive experience, after that, Borda analytical method is employed to rank the importance of each risk factor, thereby assessing project risks. This 
method synthesizes group suggestions commendably, thus people pay more and more attention to it. ${ }^{4}$

\subsection{Factor 5: benefit—mansfield model}

Mansfield model, which applies to new technologies with little information, focuses on the diffusion situation of a new technology to judge the earning power of the new technology. Factors inspected by Mansfield model are company profitability and the relationship between technological level and effect, while indexes which reflect profitability from financial angle are profit ratio of sales and return on assets. We choose total profit(profit before tax) as the profit mentioned above to calculate (See Table 3).

Table 3 - Profitability grades on debris removal technology

\begin{tabular}{|c|c|c|c|c|c|}
\hline Rank & Name & Grade & Rank & Name & Grade \\
\hline 1 & Electrodynamics Tether & 0.3629 & 4 & LODR & 0.2456 \\
\hline 2 & CubeSat & 0.2938 & 5 & Robot & 0.2441 \\
\hline 3 & Satellite & 0.2579 & 6 & Acquirer & 0.2316 \\
\hline
\end{tabular}

\subsection{Comprehensive assessment model}

\subsubsection{Entropy-weighting TOPSIS}

TOPSIS (Technique for order preference by similarity to ideal solution), a multi-objective decision method. ${ }^{5-6}$ Compared to traditional multivariate statistics method for problem assessment, TOPSIS has several characteristics such as visualized analytical principles, simple calculation and modest requirement on sample capacity.

Generally speaking, in comprehensive evaluation, the greater variation degree for a sort of index value is, the smaller information entropy is, the larger information content provided by the index is and the greater weight of the index is. Hence according to the variation degree of each index value, entropy weight, weight of each index, can be calculated by entropy ${ }^{[10]}$.

Therefore, entropy weight TOPSIS method was adopted here to establish the assessment model.

\subsubsection{Results \&Analysis}

After calculation, comprehensive evaluation values of each method in five years are respectively:

\section{$0.7442,0.7680,0.4517,0.6040,0.7821,0.6781$}

Using radar map more image expression of our results is obtained in Fig. 3.

\section{Conclusions}

When adopting our assessment indexes, we endeavor to include all important influence factors to make evaluation more precise. A scientific index system with five aspects is established to make up the shortage of previous research work, which only does comparisons between single indexes and lacks research on comprehensive competitiveness. We thoroughly solve the problem of low accuracy for evaluation result due to the uncertain weight in current AHP and fuzzy evaluation theory. We reveal the main factors which have influence on 
competitiveness of debris removal technology, and then we complete the evaluation index system and method on technologies which affect debris removal.

Although we adopt many indexes to evaluation a method, we still neglect some influence factors. Some weight allocations in weight model may not be the best options.

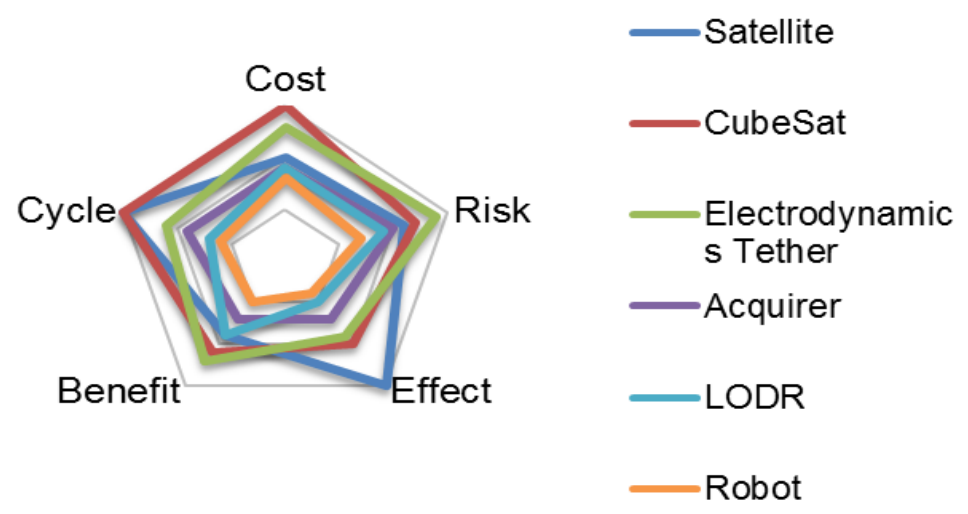

Fig.3 - Radar chart

\section{Acknowledgements}

This work is supported by Science and Technology Thinking Library Project of Liaoning Province Association for Science and Technology, Research on Enterprise Innovation Culture Construction in Liaoning Province (No.Inkx2015109). The authors also would like to thank Dr. Hai Yu and Prof. Lingling Luo for their useful discussion and assistance.

\section{References}

1. https://en.wikipedia.org/wiki/TOPSIS (2.1.2016.)

2. F. Nobuo, N. Hisaho, Monte Carlo algorithm for the double exchange model optimized for parallel computations, J. Computer Physics Communications. 142(3) (2001) 410412.

3. https://en.wikipedia.org/wiki/Analytic_hierarchy_process(1,4,2016.)

4. R. Paul, P. R.Garvey, Z. F.,Lansdowne Risk matrix: An approach for identifying assessing and ranking program risks, J. Air Force Journal of Logistics. (1998) 25.

5. V. M. Ozernoy, Choosing the "best" multiple criteria decision making method, J. INFOR. 30 (1992) 159- 171.

6. T. J. Stewart, A critical survey on the status of multiple criteria decision making theory and practice, J. OMEGA. 20 (1992) 569- 586. 